Histoire de l'éducation

$101 \mid 2004$

Varia

\title{
Michel Debré et la formation professionnelle
} 1959-1971

Michel Debré and vocational training, 1959-1971

Michel Debré und die berufliche Fortbildung, 1959-1971

Pierre Benoist

(2) OpenEdition

Journals

Édition électronique

URL : https://journals.openedition.org/histoire-education/725

DOI : $10.4000 /$ histoire-education. 725

ISSN : 2102-5452

Éditeur

ENS Éditions

Édition imprimée

Date de publication : 1 janvier 2004

Pagination : 35-66

ISBN : 2-7342-0969-1

ISSN : 0221-6280

\section{Référence électronique}

Pierre Benoist, « Michel Debré et la formation professionnelle 1959-1971 », Histoire de l'éducation [En ligne], 101 | 2004, mis en ligne le 12 janvier 2009, consulté le 20 mai 2021. URL : http://

journals.openedition.org/histoire-education/725 ; DOI : https://doi.org/10.4000/histoire-education.725

Ce document a été généré automatiquement le 20 mai 2021.

(c) Tous droits réservés 


\title{
Michel Debré et la formation professionnelle 1959-1971
}

\author{
Michel Debré and vocational training, 1959-1971 \\ Michel Debré und die berufliche Fortbildung, 1959-1971
}

\section{Pierre Benoist}

1 Michel Debré est surtout connu pour sa participation à la politique générale de la France : il a été le premier des Premiers ministres de la $\mathrm{v}^{\mathrm{e}}$ République dont il a inspiré les textes constitutionnels. Chacun connaît aussi son rôle dans la conduite de la politique algérienne de la France. On traite moins souvent de ses interventions dans le domaine de la formation professionnelle des jeunes et des adultes. Pourtant, ce sujet a constitué un de ses centres d'intérêt permanents, et il a eu l'occasion de faire adopter des textes et de faire appliquer des politiques nouvelles qui ont profondément marqué le régime français de la formation professionnelle: en 1959, par deux lois sur la promotion sociale et sur la formation des travailleurs appelés à exercer des responsabilités syndicales, puis en 1966, par la loi d'orientation et de programme sur la formation professionnelle. Après l'évocation des raisons idéologiques de l'attachement de M. Debré aux problèmes de formation, on présentera ses deux initiatives majeures de 1959 et de 1966, dont on s'efforcera ensuite de dégager les caractéristiques et la portée.

2 Républicanisme et attachement à la cohésion nationale constituent les fils conducteurs de l'action de M. Debré en matière de formation. Républicain, M. Debré était un fidèle admirateur de l'œuvre de Jules Ferry, qu'il voulait poursuivre en offrant une deuxième chance à ceux qui n'avaient pu tirer tout le profit de leur scolarité. Dans ses mémoires, il rappelle avec émotion ses souvenirs d'élève de l'école primaire et de l'un de ses maîtres qui alliait les trois visages de la nation : l'école, le service public (l'instituteur était aussi secrétaire de mairie), et l'armée (l'instituteur commandait ses élèves comme une section d'infanterie) ${ }^{1}$. Dans la pensée de M. Debré, le système de promotion sociale qu'il voulait instituer conservait les caractéristiques principales du système scolaire : une progression régulière par paliers, sur la base de cours, suivis d'une certification. 
Outre l'œuvre de justice à l'égard des individus que représentait ce projet, celui-ci avait aussi, pour objectif de sauvegarder l'unité de la Nation, toujours menacée par la constitution de groupes cumulant les privilèges et les pouvoirs. Comme beaucoup de jeunes hauts fonctionnaires qui avaient commencé leur carrière avant-guerre (il avait travaillé au cabinet de Paul Reynaud, ministre des Finances, de 1938 jusqu'à sa mobilisation en 1939), il avait très mal vécu l'affaiblissement de la France à la fin de la ${ }{ }^{\mathrm{e}}{ }^{\mathrm{e}}$ République, qu'il imputait au déficit de renouvellement des élites ${ }^{2}$. L'utilisation de l'« ascenseur social » pour garantir l'unité nationale a été évoquée à plusieurs reprises, dans ses mémoires, où il rappelle Gouvion-Saint-Cyr et sa phrase célèbre sur le «bâton de maréchal »', et où il rapporte un discours prononcé en 1960 à l'École normale supérieure de Cachan : il y évoquait l'éventualité d'une révolution sociale qui porterait au pouvoir de nouvelles élites. Pour assurer la cohésion sociale, il comptait plus sur la promotion que sur la participation, chère au général de Gaulle : «En matière sociale, plus influencé par les thèses républicaines chères à Gambetta et à Jules Ferry (qu'il me paraissait l'un et l'autre mésestimer) que par l'idéologie des catholiques sociaux dont il avait lu et médité les meilleurs écrits, je croyais - et continue de croire - aux vertus de la promotion plus qu'à celles de la participation $»^{4}$. Il n'a jamais manqué de rappeler, à ce sujet, qu'il avait créé l'École nationale d'administration, comportant un concours interne ouvert aux agents en fonction, sans condition de diplôme.

Pourtant cet enthousiasme pour la formation n'était pas nécessairement partagé par ses collègues ni par les plus hautes autorités de l'État. Ses interventions dans ce domaine semblent avoir souvent été plus tolérées que souhaitées, et on peut faire l'hypothèse qu'elles ont été une concession faite à M. Debré pour compenser les contraintes qui lui étaient imposées dans d'autres domaines.

En 1934, M. Debré soutint une thèse de doctorat à la faculté de droit de Paris, sur un sujet assez inattendu chez un juriste se destinant au Conseil d'État : l'« artisanat, classe sociale $»^{5}$. L'artisanat constitue une classe qu'il est important de soutenir pour assurer la stabilité sociale, et éviter son glissement, comme en Allemagne, vers le fascisme. Il défend aussi l'apprentissage, qui est le moyen d'assurer la pérennité de cette classe. C'est probablement par fidélité à ces positions que M. Debré déposa en 1949 une proposition de loi sur l'apprentissage dans les métiers de l'artisanat ${ }^{6}$. Ce n'était toutefois que l'amorce de son action.

\section{La cohésion nationale par la promotion sociale}

6 Dès les années 1951 et 1952, M. Debré s'est beaucoup investi dans un projet de « facultés ouvrières de culture et de technique ${ }^{7}$. Le système envisagé comprenait deux niveaux : des centres et des facultés. Les centres (de cinq à dix) prépareraient aux facultés, mais formeraient aussi des techniciens. Les facultés, au nombre de trois, formeraient des ingénieurs. Les auditeurs seraient rémunérés. Le ministre André Marie promit une commission d'étude, qui fut effectivement créée par arrêté du 21 février 1952, et dont M. Debré était membre. Elle devait recenser les résultats obtenus par le Conservatoire national des arts et métiers et les cours de perfectionnement de la promotion ouvrière. L'affaire fut résolue par le développement des centres régionaux du CNAM, créés par un décret du 25 juillet 1952. M. Debré n'a finalement pas obtenu satisfaction : le système du CNAM, très critiqué pour la lourdeur de son enseignement, l'absence de rémunération des auditeurs et ses sévères taux d'échec, ne correspondait 
qu'imparfaitement au projet de M. Debré. Mais le projet était une préfiguration de ce que ce dernier voulait réaliser par la promotion sociale: un système à niveaux successifs, n'exigeant pas de diplôme d'entrée, ouvert à ceux qui travaillent, avec conservation de la rémunération pour les périodes d'enseignement à temps plein. C'est ce que tentera M. Debré avec le projet de « Promotion sociale ».

7 Rien ne justifiait apparemment l'urgence donnée aux deux lois des 31 juillet et 28 décembre 1959 sur la promotion sociale, annoncées dans la déclaration d'investiture de M. Debré, Premier ministre, du 15 janvier 1959. La grande affaire de l'époque était la situation en Algérie. Mais il y avait aussi d'autres urgences, notamment économiques, avec l'entrée en vigueur prochaine du Marché commun. D'autre part, le programme législatif du gouvernement était très chargé : la presse de l'époque mentionnait des nombres impressionnants de textes ${ }^{8}$, parmi lesquels la promotion sociale ne figurait pas, donnant lieu seulement à des informations succinctes à quelques reprises ${ }^{9}$. Les dispositifs qui existaient dans le domaine de la formation à caractère professionnel dépendant principalement, soit du ministère de l'Éducation nationale, soit du ministère du Travail, n'étaient pas en effet, dépourvus d'efficacité, et les modifications nécessaires relevaient presque toutes du domaine réglementaire aux termes de la nouvelle constitution. L'hypothèse que nous formulons est que $\mathrm{M}$. Debré, conscient d'une demande latente de formation, réalisait un projet qui lui tenait à cœur depuis sa jeunesse, utilisant à cette fin un créneau de liberté que le Président de la République lui laissait, dans un domaine où il ne souhaitait pas intervenir personnellement.

\section{La formation professionnelle en 1959}

8 Le ministère du Travail assurait la tutelle de l'Association nationale pour la formation rationnelle de la main-d'œuvre (ANIFRMO) créée en 1949, mais dont les racines remontent à la période de l'entre-deux guerres. Cet organisme était très axé sur les formations de bâtiment et de la métallurgie, nécessaires à la reconstruction, et assurées dans 125 centres de formation. Les effectifs du personnel formé annuellement étaient passés de 11400 environ en 1947 à 25600 en $1959^{10}$. L'aspect de promotion était évident, puisque le certificat de formation professionnelle délivré à la fin du stage à de jeunes adultes sans qualification leur donnait la compétence d'ouvrier professionnel. Le ministère de l'Éducation nationale était fier de son système de "promotion ouvrière " défini par un arrêté du 15 avril 1948. Il consistait en cours d'une centaine d'heures par an, auxquels s'ajoutaient des enseignements pratiques, donnés hors du temps de travail et sans rémunération ${ }^{11}$. Le dispositif changea d'appellation vers 1954, pour s'appeler «promotion du travail » afin d'intégrer le personnel non ouvrier.

9 Le système était caractérisé par une logique d'offre : les établissements proposaient aux adultes les formations qu'ils dispensaient aux élèves. Les formations étaient du niveau de préparation au certificat d'aptitude professionnelle, plus rarement au brevet professionnel.

10 Le dispositif était couronné par la promotion supérieure du travail, organisée dans les universités, soit en instituts, soit en associations, dans quatre villes importantes, Grenoble, Nancy, Besançon et Strasbourg. Ces centres s'ajoutaient aux cinq centres régionaux du Conservatoire national des arts et métiers. La promotion du travail connaissait une évolution dynamique: de 1950 à 1959, les effectifs formés annuellement passèrent de 24440 à 130800 . Malgré ses performances apparentes, le 
système faisait l'objet d'une réflexion critique. Un fonctionnaire de l'Éducation nationale émettait en 1957 une série de critiques et de propositions ${ }^{12}$ : le système ne tenait pas suffisamment compte des besoins liés à l'évolution de l'économie, étant prisonnier des formations traditionnelles; il ne répondait pas à la demande, forte à l'époque, de formation de techniciens de niveau moyen et supérieur et il ne comportait pas de régulation interministérielle. Un an plus tard, en décembre 1958, un rapport de Jacques Wolff, réalisé pour le Commissariat général à la productivité, apportait aussi son lot de remarques et de propositions ${ }^{13}$. Il existait donc, fin 1958, une somme de réflexions et de propositions qui permettaient d'envisager une mise à niveau des dispositifs existants dans le domaine de la formation professionnelle des adultes à but promotionnel par une voie qui restait à déterminer.

11 En outre, la période était favorable à des réformes. Au cours des années 1950, de grandes évolutions marquèrent le domaine de la gestion de la main-d'œuvre et de la formation.

Ainsi, entre les années 1956 et 1959, des rapprochements s'opérèrent entre le mouvement Peuple et Culture, principalement tourné vers l'éducation populaire, et le monde du travail par l'intermédiaire des ingénieurs et cadres des entreprises publiques, d'inspiration chrétienne sociale, qui cherchaient à modifier les rapports sociaux par la formation ${ }^{14}$. Guy Hasson, responsable de la formation des Charbonnages de France, introduisit dans cet organisme la méthode de l'» entraînement mental » propre à Peuple et Culture ${ }^{15}$. En même temps, des réflexions se développaient au sein des entreprises sur la gestion du personnel. Cette fonction des entreprises, qui a longtemps été confiée au secrétariat général, prit progressivement son autonomie. Dans les années 1950, la direction du personnel devint un sujet d'étude ${ }^{16}$.

Des conceptions nouvelles dans l'appareil productif firent naître des demandes en terme de niveau de qualification de la main-d'œuvre. Pour remédier à l'insuffisance de la qualification des ouvriers, la solution mise en avant consista à développer activement la catégorie des techniciens. Le deuxième plan (1954-1957) le mentionnait expressément, et un avis du Conseil économique du 20 octobre 1953 demanda la formation de cadres techniques. Cette demande se retrouva aux journées d'études du CNPF des 11 et 12 juin $1955^{17}$.

\section{Les lois de 1959}

La loi du 31 juillet 1959 relative à diverses dispositions tendant à la promotion sociale ${ }^{18}$ fut votée très rapidement. Venue en discussion le 30 juin 1959, elle fut promulguée le 31 juillet et publiée le 6 août. Elle avait donné lieu à la procédure officielle créée par les nouvelles institutions d'une commission spéciale dont le rapporteur était André Fanton, ancien membre du cabinet de M. Debré au ministère de la Justice et récemment élu député.

15 Si l'on s'en réfère aux mémoires de $\mathrm{M}$. Debré ${ }^{19}$, ce dernier se serait attelé à la tâche à la fin du mois de janvier 1959 avec Pierre Racine, Jérôme Monod et Jacques Decoust. Bertrand Schwartz, directeur de l'École des Mines de Nancy, lui aurait fait part de ses expériences en matière de formation des adultes. En fait, ce fut J. Decoust qui tint la plume dans ce projet. Cet inspecteur du travail exerçait aux Charbonnages de France quand M. Debré obtint qu'il fût placé auprès de lui, avant de le nommer délégué général 
à la Promotion sociale. Quant à B. Schwartz, son intervention dans les affaires de promotion sociale est postérieure à la mise en œuvre de la loi ${ }^{20}$.

Les idées transcrites dans le projet étaient des idées personnelles de M. Debré. Les projets sociaux du RPF n'ont pas eu d'influence sur ses réflexions. Quant à l'attitude du général de Gaulle à l'égard de ce projet, elle consistait à laisser agir le Premier ministre, l'affaire étant de sa compétence et faisant même partie de ses passions personnelles ${ }^{21}$.

La modestie volontaire des objectifs affichés apparaît dans le titre: "diverses dispositions tendant à la promotion sociale ». Cette rédaction prudente s'explique par le souci de calmer les inquiétudes des ministères formateurs et notamment des milieux de l'Éducation nationale qui ressentaient le projet de M. Debré comme une dépossession d'une attribution importante. Le texte reprenait à quelque chose près le dispositif existant. Il renvoyait à des décrets l'organisation d'un niveau de formation des techniciens et du personnel d'encadrement. Il spécifiait des objectifs pour l'agriculture, pour l'artisanat, mentionnait les entreprises et écoles privées parmi les organisateurs des cours, recommandait la passation de conventions, sans les rendre obligatoires. La promotion supérieure était reprise du projet de facultés ouvrières, en généralisant les expériences de Grenoble notamment. Étaient proprement innovantes les dispositions qui prévoyaient une rémunération des stagiaires qui ne sera que parcimonieusement accordée, la prise en charge financière par la taxe d'apprentissage, et surtout la mise en place d'un dispositif interministériel de coordination. Ce texte mettait au premier rang de ses objectifs des préoccupations sociales, mais n'omettait pas les besoins de l'économie. Un effort particulier fut fait pour mettre en valeur les réalisations existantes, tant étaient vives les incompréhensions de cette loi du côté de l'Enseignement technique, à propos de la coordination interministérielle dont l'introduction dans le texte fut prudemment laissée à l'initiative du rapporteur André Fanton; ce dernier traita aussi de l'objectif de cohésion nationale, en dénonçant l'attrait qu'exerçait sur une partie importante de l'électorat l'idéologie propagée par le Parti Communiste.

Dans l'ensemble, le climat était plutôt à un scepticisme poli, même dans la majoritéé2 Dans les milieux politiques, et au Parlement en particulier, les critiques les plus vives provinrent du groupe communiste qui déclarait craindre que cette politique ne servît d'alibi à une réduction des ressources attribuées aux établissements de formation initiale. Parmi les partenaires sociaux, la puissante Union des industries métallurgiques et minières fit des remarques d'organisation, qui tendaient à réduire les cas de maintien du salaire. La CGT regretta une utilisation plus intensive des moyens de formation qui dispensait de les augmenter, un centrage prioritaire sur les cadres au détriment des jeunes sans formation professionnelle, un pouvoir discrétionnaire des chefs d'entreprise dans le choix des personnes admises en stage. La CGT-FO et la CFTC mentionnèrent la parcimonie des moyens dégagés et le caractère exclusivement professionnel donné à la promotion sociale, qui demanderait une politique plus large et plus ambitieuse. Quant au Centre des jeunes patrons, il adhéra à l'objectif de promotion sociale, mais il privilégiait la promotion collective par rapport à la promotion individuelle ${ }^{23}$.

\section{La loi du 28 décembre 1959}

19 La presse et l'opinion publique se sont, à tort, beaucoup moins intéressées à ce texte qu'à celui qui traite de la promotion des salariés. Cette loi organisait la formation des 
cadres syndicaux soit dans des centres dépendant des organisations syndicales les plus représentatives, soit dans des instituts d'université ou de faculté. L'État apportait une aide financière par des crédits affectés au ministère du Travail et au ministère de l'Éducation nationale. Une des intentions du projet était de renforcer la capacité de discussion en matière économique des organisations syndicales, objectif qui avait déjà été servi par la loi du 23 juillet 1957 sur le congé-éducation, sans prévoir toutefois de rémunération: si les centrales syndicales étaient dotées, certaines depuis l'avantguerre, de centres de formation pour leurs responsables, l'aide extérieure était parcimonieuse : fonds issus de la Charte du travail de Vichy, fonds provenant de l'aide américaine et se divisant en une partie banalisée et une partie provenant de l'amendement Blair-Moody (1952) qui liait développement du syndicalisme et libreentreprise, et, de ce fait fut refusée par la CGT. La loi du 28 décembre 1959 permit de financer les quatre Instituts du travail de Lille, Strasbourg, Grenoble et Aix-enProvence et le Centre de culture ouvrière de Paris, qui non seulement assuraient un enseignement, mais indemnisaient les stagiaires, et aussi de subventionner les centrales syndicales à l'exception de la $\mathrm{CGT}^{24}$. Cette dernière, tenue en suspicion pour risque de "débordement politique », selon l'expression de Gilbert Grandval, ministre du Travail, lança une offensive en avril et mai 1964 pour être admise à la subvention ${ }^{25}$, ce qui ne fut acquis qu'en 1968 .

\section{Les réalisations}

En 1964, Bernard Chenot, dans le rapport présenté au nom de la Commission d'étude des problèmes de formation et de promotion sociale en février 1964, proposa la définition suivante de la Promotion sociale: "Doit être considérée comme action de promotion sociale toute action de formation ou de perfectionnement, de quelque nature qu'elle soit, à quelque niveau qu'elle se situe, dès lors qu'elle s'adresse à une personne déjà engagée dans la vie professionnelle et qui contribue à un avancement dans l'échelle sociale ».

21 Une définition aussi large permettait d'englober en fait toutes les actions de formation. Aussi, plutôt qu'un bilan, on ne peut en donner qu'un simple aperçu. Un Comité de coordination fut institué auprès du Premier ministre et sous sa présidence. Il formulait des propositions, donnait des avis sur les projets de textes, sur l'usage des moyens. Une Délégation générale à la promotion sociale fut créée par décret du 29 mai 1961 et confiée à J. Decoust. Le Fonds de la promotion sociale était constitué par un chapitre du budget des services du Premier ministre. Des comités départementaux (45 au premier janvier 1966) et des comités régionaux (5), présidés par les préfets et rassemblant des représentants des partenaires sociaux étaient chargés de recenser les besoins et de faire des propositions.

22 Les crédits de promotion sociale, tous ministères confondus, passèrent de 149,80 MF en 1960 à 311,00 MF en 1965, auxquels il convient d'ajouter respectivement 1,5 et $80 \mathrm{MF}$ de crédits d'équipement du ministère du Travail. Le plus important bénéficiaire était le ministère du Travail dont la dotation passa de $128 \mathrm{MF}$ à $225 \mathrm{MF}$, mais elle englobait toute la subvention des centres de FPA. Hormis ce cas exceptionnel, les dotations des autres ministères furent plus modestes et concernèrent essentiellement l'Éducation nationale, l'Agriculture et les Armées. Les effectifs des auditeurs atteignirent 453000 en 
1965, dont 306000 relevant de l'Éducation nationale, laquelle comptabilisait d'ailleurs l'enseignement par correspondance ${ }^{26}$.

Le coefficient d'accroissement des effectifs de la promotion sociale fut de $22 \%$ par an. Celui de la promotion du travail, qui le précédait, de $48 \%$. Ceci relativise considérablement la valeur ajoutée à attribuer au nouveau dispositif. Par niveau de qualification, les plus grands bénéficiaires furent les ouvriers et employés qualifiés et les cadres, à l'exclusion des personnes sans qualification. La promotion collective (formation des cadres syndicaux) passa de 25600 personnes en 1962 à 52800 en 1965, ce qui constitue indéniablement une réussite.

Les ministères tirèrent diversement parti des nouvelles dispositions.

\section{L'Éducation nationale}

Une longue circulaire du 22 décembre 1959 rappela les réalisations déjà atteintes, et décrivit les nouvelles dispositions. Elle créa un échelon central de la promotion sociale auprès du ministre confié à un inspecteur principal de l'enseignement technique qui deviendra inspecteur général de l'instruction publique, M. Cercelet. Dans chaque académie, un inspecteur chargé des "enseignements complémentaires" fut désigné auprès de l'inspecteur principal de l'enseignement technique. Dans chaque cours de promotion sociale devait être créé un conseil de perfectionnement regroupant administration, enseignants, employeurs et auditeurs. Les effectifs accueillis étaient essentiellement masculins : selon une note du 10 février 1964, dans les cours de $1^{\mathrm{er}}$ et $2^{\mathrm{e}}$ degrés (concernant les ouvriers spécialisés et les ouvriers qualifiés) les femmes étaient au nombre de 37000 sur un effectif total de 170000 . Au CNAM, on comptait 6000 femmes sur 40000 auditeurs.

Le financement comportait la taxe d'apprentissage, des subventions des collectivités locales et une subvention de l'État, attribuée par le ministère après avis du comité départemental de l'Enseignement technique et de l'Inspection générale et souvent proche de la demande ${ }^{27}$. Parmi les organismes subventionnés par le ministère, on relève principalement des établissements scolaires, des cours municipaux, des associations professionnelles ou compagnonniques, des chambres des métiers. Les formations sollicitées étaient principalement du niveau du certificat d'aptitude professionnelle, avec poursuite vers le brevet professionnel ${ }^{28}$. Le système fit l'objet de critiques : il lui était reproché de fonctionner à guichet ouvert, comme s'il suffisait de présenter un projet pour qu'il fût accepté, l'attrait des indemnités accordées au chef d'établissement provoquant une inflation des cours ${ }^{29}$. On considérait comme d'excellents résultats des scores de 29 reçus sur 53 présents à un brevet professionnel de mécanique, ce qui donne une idée de la difficulté des formations en cours du soir. L'organisation était très centralisée, tout se décidant à l'administration centrale, cours par cours.

M. Debré imposa en outre avec ténacité la mise en place d'une voie promotionnelle d'accès aux grandes écoles réservée aux élèves de l'enseignement technique, à laquelle il attachait une valeur symbolique; malheureusement, l'institution ne la soutint guère après son départ ${ }^{30}$.

\section{Le ministère du Travail}

28 Le nombre des stagiaires formés par l'ANIFRMO augmenta peu pendant les premières années de la promotion sociale : 25600 en 1959, 23800 en 1962. Ce n'est qu'en 1963 que 
les effectifs de candidats reçus crurent régulièrement pour atteindre 40600 en $1968^{31}$. La nouvelle législation permettait pourtant d'améliorer la rémunération des stagiaires. Quant à la formation continue, 513 stagiaires seulement sont repérés sous cette rubrique en 1960, et 1749 en $1970^{32}$.

\section{Le ministère de l'Agriculture}

La formation des adultes dans un but promotionnel ne datait pas de 1959. Jean-François Chosson a décrit l'histoire de cette formation ${ }^{33}$. Elle a commencé avant la guerre et a mobilisé d'importants mouvements d'éducation populaire. La "vulgarisation » a été organisée par un décret du 11 avril 1959, contemporain de la loi sur la promotion sociale. Des dispositions ont été prises pour lier formation des jeunes et accès à l'exploitation : un décret du 14 juin 1961 donne un avantage d'un an sur le délai requis pour l'installation au bénéfice des jeunes qui justifient d'une formation professionnelle. On peut citer, parmi les réalisations, la création de centres pour faciliter la reconversion de la main-d'œuvre agricole excédentaire à Tinténiac, Redon (Ille-etVilaine), Josselin (Morbihan), Pont-de-Bris (Finistère) avec une forte participation financière de la Délégation ${ }^{34}$. La promotion dite collective, celle qui concerne les militants des organisations professionnelles, fut particulièrement active en agriculture.

\section{Le ministère des Armées}

30 Un comité de travail pour la promotion sociale fut mis en place à compter du $1^{\mathrm{er}}$ juin 1962 sous la présidence du général Gambiez. Le livre blanc de la Promotion sociale mentionne l'existence de 1000 officiers-conseils, de 10000 jeunes présents dans 260 clubs agricoles. Il est fait état de 23000 soldats pris en charge au niveau du certificat d'études primaires en 1966, et de 12000 à un niveau inférieur, de 1500 clubs de loisirs éducatifs, et de l'existence d'un centre inter-armées de formation d'animateurs (CIFA) à Angoulême ${ }^{35}$. L'enseignement à distance était mis à contribution. Les publics visés en priorité étaient ceux des jeunes agriculteurs menacés de conversion forcée du fait de la modernisation de l'agriculture. Le bilan 1965-1966 confirme ces chiffres et précise que les jeunes avaient acquis 1945 certificats d'études primaires, 1105 certificats d'aptitude professionnelle, et que les entrées en formation professionnelle des adultes avaient été de $1666^{36}$.

\section{Les maisons de la Promotion sociale}

Il s'agissait d'offrir des salles de cours et des moyens d'hébergement pour les stagiaires et les organisations, voire d'assurer un certain soutien logistique aux organisations qui en manquaient. Mais d'autres développements étaient possibles. La création de centres de promotion sociale mobilisa près de la moitié de la dotation du Fonds en 1963. En 1966, le Fonds finança les centres de Chambéry, Bordeaux, Grenoble et Gap ${ }^{37}$. Une étude particulière à la Maison de Grenoble a été réalisée sur la période 1960-1966 $6^{38}$. La réussite de ces institutions a été incertaine ; lors de la mise en place de la loi de 1966, le programme d'action mentionnait la nécessité d'une clarification du rôle de ces organismes ${ }^{39}$. 


\section{Autres réalisations} difficultés d'organisation pédagogique et administrative inhérentes à toute tentative d'envergure dans le domaine de la formation des adultes. L'organisation pédagogique et administrative des cours se heurtait à des difficultés réelles, largement dénoncées, pour la plupart à la suite des rapports Chenot et Masselin et aussi révélées par les documents d'archives ${ }^{43}$. Les stagiaires étaient d'un niveau trop bas et trop hétérogène, on manquait de méthode pédagogique adaptée à leur cas, de matériel pédagogique (on se préoccupa par ailleurs de créer des manuels spécifiques) et les taux de réussite aux examens étaient faibles. On s'interrogea aussi sur la pertinence de l'examen traditionnel pour sanctionner la formation. L'année scolaire n'était pas un cadre approprié, car pendant les vacances, les stagiaires perdaient leurs acquis. La rémunération du personnel était un sujet de revendication. Les critiques concernèrent principalement les problèmes d'horaire et de rémunération des stagiaires. Le fait d'effectuer la grande majorité des formations en dehors des heures de travail était générateur de fatigue, désorganisait la vie familiale et compromettait les résultats. C'est ainsi que les stagiaires qui bénéficiaient, grâce au temps partiel, de facilités d'horaires avaient un taux de fréquentation de 90 à $95 \%$, et des succès au BP et au BEI de l'ordre de 60 à $70 \%$, alors que les autres avaient un taux de fréquentation de $50 \%$ et une réussite de l'ordre de 15 à $20 \%$. Ce fut un des points importants du rapport de la Commission Chenot que de demander pour tous une prise en charge de la formation pour moitié dans le temps de travail. Les indemnités pour perte de salaires, pourtant prévues par la loi, ne furent accordées que pour la promotion supérieure, et réservées à la troisième année.

L'interministérialité a été le point d'achoppement principal de l'organisation. Le délégué général, quelles que fussent ses qualités, n'avait que l'autorité que lui prêtait le Premier ministre. Après le départ de $\mathrm{M}$. Debré du gouvernement, le délégué s'est retrouvé «orphelin », le nouveau Premier ministre, G. Pompidou ne prêtant pas à la promotion sociale le même intérêt que son prédécesseur. Ces querelles de territoire ont trouvé leur lieu d'expression privilégié dans la procédure budgétaire : la Délégation devait donner un avis sur l'ensemble des crédits concernant la promotion sociale dont les ministères demandaient l'inscription à leur budget; mais cette règle a toujours rencontré des résistances, les plus vives venant du ministère du Travail, qui ne voulait pas faire entrer dans l'enveloppe de la promotion sociale les crédits destinés aux centres de $\mathrm{FPA}^{44}$. D'ailleurs, M. Debré se déclara décidé, après son départ de Matignon 
et dans l'éventualité d'un retour au gouvernement, à créer un nouveau ministère de " formation technique ", en retirant cette fonction aux ministères chargés du Travail et de l'Éducation, notamment. Néanmoins, un objectif fut atteint : la formation des adultes n'était plus de la compétence exclusive de quelque ministère et ne le sera plus jamais. La conception même du dispositif souffrait aussi d'ambiguïtés. La première concerne l'exclusion de l'éducation populaire d'un dispositif tendant à assurer la promotion sociale. Ce choix est d'autant plus étonnant que les mouvements d'éducation populaire avaient connu un grand essor après la Libération. D'après certains témoins et acteurs, ce clivage serait un phénomène commun à tous les pays évolués ${ }^{45}$. Selon A. Fanton, il était de l'intention du gouvernement et du Parlement de laisser l'éducation populaire aux soins des associations. En fait, il semble que l'éducation populaire n'entrait pas dans les limites de l'épure que s'était fixée le Premier ministre. Son objectif était de constituer un dispositif très structuré. Dans un tel système "parascolaire ", il n'y avait guère de place pour des formations moins normées et aux objectifs très variés. En quelque sorte, l'éducation populaire n'était pas « administrable » comme peut l'être un système proche du système scolaire. Les mouvements d'éducation populaire purent néanmoins trouver des moyens de développement appréciés auprès du HautCommissaire à la Jeunesse et aux Sports, Maurice Herzog ${ }^{46}$.

La seconde affectait la notion même de promotion sociale : certains auteurs affirment que la promotion sociale n'a jamais prospéré et qu'elle régressa même chez les ouvriers non-qualifiés et les ouvriers qualifiés, surtout entre 1965-1970 et 1988-1993 ${ }^{47}$. L'idée même de promotion sociale serait en voie de disparition, les salariés, sensibles à un modèle des "compétences " ayant désormais des aspirations individuelles de prise en charge de leur propre parcours qui ne ferait plus nécessairement appel à des formations : la société promotionnelle ne se réalisait pas et la loi aurait été la marque d'une pensée tournée vers le passé ${ }^{48}$. Il est vrai que les idées de 1959 étaient encore proches de celles de la Libération, époque à laquelle la notion de « promotion » était à l'ordre $\mathrm{du}$ jour. Il se trouve que l'accélération des évolutions économiques a progressivement privilégié l'adaptation aux techniques par rapport à la promotion des individus. Dans les faits, les termes de "promotion sociale" sont vite devenus synonymes de «formation professionnelle ». Dès 1963, le responsable de la promotion sociale à l'Éducation nationale exposait devant la commission Chenot les missions attendues du système de promotion sociale : le rattrapage, la promotion, la mise à jour, la reconversion. C'était déjà le catalogue des actions de la loi de 1971.

Le fait déterminant est que, dès 1960, l'activité économique s'accéléra: alors que de 1955 à 1959, la croissance du PIB était de 4 \% par an, elle passa à 7,3 \% de 1959 à 1965 $(\text { prix } 1970)^{49}$ : l'envolée de l'économie a dramatisé le problème de la formation et balayé les préoccupations de promotion des individus.

Le projet de $\mathrm{M}$. Debré ne s'est donc pas réalisé de la manière dont il l'avait prévu, mais il a constitué le socle de développements ultérieurs : la formation professionnelle était désormais l'objet d'une loi, même si le domaine n'était pas législatif. Ainsi a été créé un précédent qui a ouvert la voie aux textes de 1966 et de 1971. D'autre part, un financement spécifique a été attribué à la formation des adultes: le fonds de la promotion sociale s'est pérennisé sous les lois ultérieures. Enfin une capitalisation des compétences techniques en matière de formation d'adultes s'est progressivement constituée: des personnes se sont qualifiées dans des fonctions nouvelles, des 
connaissances se sont accumulées et ont été réinvesties. Les lois de 1959 ont marqué les premiers pas d'une reconnaissance de la formation continue.

\section{Le lancement de la formation professionnelle : 1966}

40 Le 8 janvier 1966, M. Debré est ministre de l'Économie et des Finances dans le gouvernement de Georges Pompidou. Son directeur de cabinet, Antoine DupontFauville, lui avait déconseillé de reprendre du service comme simple ministre ${ }^{50}$, mais il avait fini par accepter, tout en revendiquant une place privilégiée dans le gouvernement, notamment en exerçant une sorte de tutelle sur les ministères économiques et sociaux, place que le Premier ministre n'avait pas osé lui refuser, mais qui créait une situation assez malsaine ${ }^{51}$. C'est dans ce cadre institutionnel assez insolite que M. Debré eut l'opportunité d'intervenir à nouveau dans le domaine de la formation professionnelle.

\section{Origine et élaboration de la loi du 31 décembre 1966}

M. Debré avait demandé à Noël 1965 à Antoine Dupont-Fauville de lui proposer un programme d'action dès le début de l'année. Celui-ci lui proposa trois thèmes: l'éducation et la formation, les problèmes fonciers et le logement, la recherche ${ }^{52}$. En tant que ministre de l'Économie, M. Debré pouvait être habilité à intervenir dans ces domaines qui conditionnaient le développement de l'activité économique. En outre, M. Debré était connu pour sa passion envers ce domaine, alors que G. Pompidou ne lui manifestait qu'un intérêt minime. On retrouvait donc une situation comparable à celle de 1959: M. Debré s'est vu concédé un domaine réservé pour lequel il avait une appétence personnelle. A. Dupont-Fauville avait fait appel à Dominique Alunni, ancien ajusteur-outilleur, ancien militant de la CFDT, ancien président de la Jeunesse ouvrière chrétienne, président de l'Institut national de formation des animateurs de collectivités (INFAC) pour apporter des idées à l'élaboration de la loi, à titre officieux ${ }^{53}$.

L'époque était favorable à une reprise des actions sur la formation. Henri Mendras date de 1965 la rupture avec la période de l'après-guerre ${ }^{54}$. Le Premier ministre lançait le thème de l'industrialisation, un courant moderniste animait le CNPF avec Ambroise Roux à la présidence de la commission économique, François Bloch-Lainé plaidait pour un nouveau management de l'entreprise ${ }^{55}$. La mise en œuvre du marché commun exigeait une compétitivité accrue de l'économie française ; le V Plan avait mis l'accent sur le besoin de formation du personnel à tous les niveaux. Ces objectifs furent nettement mis en avant dans la présentation que fit M. Debré du projet de loi devant l'Assemblée nationale le 5 octobre 1966. M. Debré reprenait ainsi un de ses thèmes de prédilection. Dans ses mémoires ${ }^{56}$, il mentionne avec une certaine amertume le fait que la tâche qu'il avait entreprise en 1959 sous l'appellation de promotion sociale avait été interrompue après son départ. Le 31 janvier 1966, un chargé de mission au cabinet dressa, dans une note pour le ministre sur l'éducation permanente, un bilan pessimiste des efforts faits de 1959 à 1965 : les besoins n'étaient pas couverts, il n'existait pas de doctrine d'ensemble, les structures de coordination n'avaient pas fait la preuve de leur efficacité. Il concluait à la nécessité de repenser le problème dans son ensemble, voire 
de saisir le Conseil économique et social, et posait la question d'une déclaration d'intention du gouvernement ${ }^{57}$.

Comme en 1959, la nécessité d'une loi pouvait se discuter. M. Debré la justifia, dans son discours de présentation, d'une part par l'obligation nouvelle qu'il voulait instituer pour l'État, les personnes morales de droit public, les entreprises et les organisations professionnelles et par la programmation des dépenses publiques. P. Laurent, qui fut commissaire du gouvernement pour présenter le projet de loi au Conseil d'État, se souvient avoir eu du mal à convaincre ses collègues de la nécessité d'une loi. En fait, les archives montrent que la paternité de la loi revient bien à $\mathrm{M}$. Debré, et non aux fonctionnaires du Plan, comme il a pu être soutenu ${ }^{58}$. M. Debré avait décidé dès février 1966 de faire une loi-programme : une note du $1^{\text {er }}$ mars 1966 de Françoise Duléry, membre du cabinet, relatait un entretien du ministre avec Pierre Laurent, secrétaire général du ministère de l'Éducation nationale, le samedi 26 février : le ministre voulait faire une loi-programme sur trois ans en matière de formation et d'enseignement professionnel. On pourrait insérer dans cette loi des dispositions relatives à l'organisation régionale (le ministre était frappé par l'absence de coordination de la formation professionnelle à ce niveau), et à la réorganisation du système des bourses pour les étudiants âgés, pour les femmes et pour le recyclage des cadres.

Pierre Laurent pensait que le projet devait sauvegarder la personnalité de l'enseignement technique, que le système de coopération avec les professions était à revoir, que l'organisation régionale de la formation professionnelle était souhaitable si elle était présidée par le recteur ; il mentionnait aussi la généralisation des conventions avec les professions et la réforme de la taxe d'apprentissage ${ }^{59}$. Une note du cabinet Debré du 29 avril relate le contenu d'une réunion tenue la veille chez le ministre de l'économie et des finances. Elle met l'accent sur la coordination, le rôle des conventions, les priorités (pour l'Éducation nationale, l'enseignement technique, pour les affaires sociales, l'implantation des centres de FPA en fonction des besoins économiques, l'augmentation de la taxe d'apprentissage de 0,4 à $0,6 \%{ }^{60}$. Une structure légère fut mise en place pour préparer la loi, dont la responsabilité opérationnelle incombait à Françoise Duléry et à Pierre Laurent sous la présidence de M. Debré, mais le groupe se réunissait aussi en son absence.

Dans les archives du Commissariat général du plan, on trouve certes trace des réflexions qui y furent menées sur les problèmes d'adéquation de flux de formation et de besoins de main-d'œuvre, mais en revanche, aucune mention de la demande d'une loi nouvelle. Les archives comportent en outre une note d'analyse du premier projet de loi par les services du Plan, qui montrent que ces derniers découvrirent avec étonnement les dispositions proposées ${ }^{61}$.

La procédure d'adoption de la loi fut, comme en 1959, marquée par l'urgence. La Commission des affaires culturelles, familiales et sociales protesta contre les délais qui lui étaient imposés : adopté en Conseil des ministres le 28 septembre, le texte vint en discussion à la Commission des Finances, de l'Économie générale et du Plan de l'Assemblée le 14 septembre, au Conseil économique et social le 20, à la Commission des Affaires culturelles, familiales et sociales le 30 . Votée en première lecture par l'Assemblée nationale le 6 octobre, elle fut discutée et votée par le Sénat le 27 octobre, puis par l'Assemblée en deuxième lecture le 18 novembre. Des interventions pressantes de $M$. Debré avaient été nécessaires auprès de Christian Fouchet, ministre de l'Éducation nationale, pour que ce dernier assistât à la séance du Conseil économique et 
social ; ses réticences étaient motivées par la crainte d'être entraîné dans un débat sur l'orientation scolaire et universitaire ${ }^{62}$, problème à propos duquel il était en désaccord avec l'Élysée. L'exposé des motifs reprenait les considérations exposées plus haut; il mettait sur le même plan formation des jeunes et formation des adultes. Il lançait officiellement un nouveau vocable : celui d'éducation permanente.

\section{Le contenu de la loi}

\section{Une obligation morale}

L'article premier déclarait que la formation professionnelle constituait une obligation nationale. Cette déclaration de principe de M. Debré - «peut-être ce à quoi je tenais le plus $»^{63}$ - devait porter la loi de 1966 au même rang que la loi de 1880 par laquelle J. Ferry avait institué l'obligation scolaire. Pourtant, le dispositif mis en place n'eut pas la même rigueur que l'ensemble législatif institué de 1880 à 1889. Les obligations imposées à cette époque étaient précises : les parents devaient envoyer leurs enfants à l'école sous peine de sanctions pénales et, symétriquement, les communes devaient ouvrir des écoles, le préfet pouvant procéder à l'inscription d'office de la dépense au budget de la commune.

L'obligation créée par la loi de 1966 n'était pas de même nature. Elle ne comportait pas de contrainte à l'égard des individus. En sens inverse, en disposant que l'État, les collectivités locales, et un certain nombre d'organismes énumérés devaient concourir à assurer cette obligation, la loi n'était pas assez précise pour permettre à un administré d'exercer des recours contentieux. Il s'agissait d'une obligation purement morale. Lors de l'examen du projet par l'assemblée générale du Conseil d'État le 27 septembre 1966, personne ne soutint que l'article premier posât une règle de droit. Pour maintenir néanmoins cette disposition dans le texte, le président estima que « on ne peut refuser d'inclure des principes pour les rendre plus affirmés $»^{64}$. Au Sénat, Michel Kistler, rapporteur de la Commission des finances, exprima la même idée dans son rapport le 27 octobre 1966: « Notons toutefois que cette affirmation solennelle de l'obligation de la formation professionnelle ne comporte aucune sanction ».

\section{Une politique coordonnée et concertée}

49 La loi prévoyait dans son article 3 que « la formation professionnelle et la promotion sociale font l'objet d'une politique coordonnée et concertée, notamment avec les organisations représentatives des employeurs et des travailleurs salariés ainsi que des travailleurs indépendants, selon des modalités qui seront fixées par décret \%. Le rapporteur du projet au Conseil d'État, Bernard Jouvin constata que les dispositions concernant la politique coordonnée et concertée étaient parmi les rares dispositions à caractère législatif $\mathrm{du}$ texte; elles auraient mérité une définition plus précise $d u$ contenu de ces deux termes, mais il s'agissait plus de pratique politique que de droit. Un conseil national de la promotion sociale, de la formation professionnelle et de l'emploi rassemblait les représentants des pouvoirs publics et des organisations professionnelles et syndicales et remplaçait à la fois le Haut Comité de l'orientation et de la formation professionnelle et le Comité de coordination de la promotion sociale. Un comité interministériel présidé par le Premier ministre et dont le ministre de l'Éducation nationale était le vice-président, réunissant les ministres intéressés, serait le lieu de confrontation des programmes, d'établissement des directives et de 
répartition des moyens financiers. Un groupe des hauts fonctionnaires dont la loi précisait qu'il était présidé, par délégation du Premier ministre, par le secrétaire général du ministère de l'Éducation nationale, préparait les travaux du conseil et, dans les faits, gérait le dispositif. Des comités régionaux étaient institués, présidés par le préfet, le recteur étant vice-président de droit. Ces dispositions furent introduites au cours de la procédure parlementaire. Enfin, un conseil de gestion du fonds de la formation professionnelle et de la promotion sociale, qui remplaçait le fonds de la promotion sociale, était présidé par un haut fonctionnaire et aurait pour rôle de proposer au comité interministériel les orientations à donner aux allocations de moyens. Si les conventions entre l'État et les centres de formation d'origine publique ou privée étaient déjà prévues par la loi de 1959, elles avaient été utilisées de manière occasionnelle. La loi de 1966 faisait de la convention le mode normal d'attribution des aides financières de l'État. Cette obligation des conventions deviendra une règle permanente de la formation continue.

La coordination administrative avait été le principal point faible du système de la promotion sociale. Le problème de la coordination fut largement évoqué lors de la discussion du projet de texte au Sénat: M. Debré le déclara lui-même le 27 octobre: "c'est sur la coordination que la loi sera jugée " $^{65}$. André Morice, faisant état de son expérience d'ancien secrétaire d'État à l'Enseignement technique, décrivit les services compétents des divers ministères comme « un ensemble disparate qui [...] interdit toute formation professionnelle cohérente ». Il ne voyait comme remède que de désigner « une personnalité siégeant obligatoirement au conseil des ministres - cela est essentiel pour son autorité et ses arbitrages - qui serait exclusivement chargée auprès du premier ministre de la coordination des activités de formation professionnelle et de promotion sociale». Ce ne fut pas la solution retenue, mais le rôle imparti à Pierre Laurent s'inspira fortement de cette conception.

51 La place prépondérante faite à l'Éducation nationale dans le dispositif s'explique d'abord par l'aspect financier de la loi: son but initial était de programmer des dépenses d'équipement, et sur les 2 milliards prévus pour les trois années 1967, 1968 et 1969, 1,3 étaient réservés aux instituts universitaires de technologie et les collèges d'enseignement technique, 0,315 à l'enseignement agricole et 0,385 aux centres de FPA ; la loi de 1966 était autant tournée vers la formation initiale, et notamment celle de l'Éducation nationale, que vers la formation continue. Pierre Laurent a pu jouer de sa position particulière dans les affaires de formation : membre du Conseil d'État, il n'était pas suspect de nourrir des préjugés pour ou contre l'un ou l'autre des ministères formateurs. Son expérience de directeur général de la main-d'œuvre au ministère chargé du Travail lui avait permis, d'une part, de mesurer la faible qualification d'une grande partie des travailleurs, mais aussi de connaître l'Éducation nationale du dehors : ses jugements sur l'institution étaient très critiques : une certaine arrogance qui portait ses représentants, notamment les inspecteurs généraux de l'enseignement technique, à décrier les formations données dans les centres de FPA; un mauvais ciblage de ses formations, qui conduisait à mettre sur le marché du travail des jeunes, notamment les jeunes filles, dotés de diplômes sans débouchés. Elle redoutait aussi la confrontation et répugnait à faire appel à des professionnels pour assurer les enseignements.

Après 1963, le secrétaire général réussit à s'imposer dans le ministère, à réaliser une réforme de structure qui confirma la disparition des grandes directions verticales, et à contrôler l'ensemble de la maison. Il mesurait l'ampleur des moyens dont disposait le 
ministère, aussi bien en personnel de formation qu'en machines-outils. La mise en œuvre de la réforme de l'enseignement de 1959 avait conduit à une réforme de l'enseignement technique. La création des instituts universitaires de technologie fut décidée, et la loi, précisément, programmait leur financement. Il était donc bien placé pour revendiquer pour l'Éducation nationale le leadership de la formation professionnelle dans sa version $1966^{66}$. Cette opération n'était pas gagnée d'avance, car M. Debré aurait souhaité la création d'un "ministère de formation technique " regroupant l'enseignement technique, la FPA et l'éducation permanente. Mais ce projet fut repoussé par G. Pompidou ${ }^{67}$. P. Laurent se déclara hostile à ce projet, car il y voyait un risque de dévalorisation des formations techniques et une occasion manquée de faire évoluer l'Éducation nationale. Il s'en expliqua le 6 septembre 1966 dans un exposé devant les représentants de la presse sur le projet de loi adopté par le Conseil des ministres le 10 août ${ }^{68}:$ " Le gouvernement a entendu tout d'abord récuser des solutions dépassées ou de fausses solutions. L'éventuel regroupement, au sein d'un organisme administratif distinct de toutes les responsabilités de formation professionnelle, est à inscrire au nombre des solutions dépassées et dangereuses. Il dresserait, en effet, un obstacle insurmontable devant l'orientation des jeunes et rejetterait les objectifs de formation professionnelle hors de l'enseignement, allant ainsi à l'encontre d'un puissant effort de rénovation et de réforme [...]. D’autre part, réserver à un organisme administratif nouveau, étranger à la fois à tous les départements ministériels intéressés, la seule fonction de coordination, c'eût été l'exposer rapidement à l'échec, faute de pouvoirs et de responsabilités directes à l'égard soit de l'enseignement, soit du marché du travail, soit des activités industrielles, commerciales et artisanales ».

La place faite au secrétaire général fut soigneusement mesurée : «le Premier ministre n'avait pas l'intention de livrer le dispositif à l'Éducation nationale. Il prit de multiples précautions en ce sens (mécanisme de gestion du fonds, désignation de Laurent parce qu'il n'était pas un enseignant) $»^{69}$. La question de la place de l'Éducation nationale dans le dispositif réapparut à propos de la désignation des secrétaires des organismes créés par la loi. Une tendance notamment partagée par le ministre chargé de la réforme administrative, Louis Joxe, était de constituer un secrétariat commun; mais le risque était de le confier à un proche de Pierre Laurent (Gabriel Ducray) ${ }^{70}$. Une note de Françoise Duléry, non datée, mentionne qu'Édouard Balladur «craint la force que représente Laurent ; d'autre part, il ne veut pas instaurer la pagaille ». La position de F. Duléry était la suivante : « Laurent coordonne, mais il ne peut réussir que s'il le fait au nom du premier ministre "; en outre, elle était favorable à ce que le secrétariat du conseil du fonds et du conseil national fût léger et non autonome par rapport à $\mathrm{P}$. Laurent ${ }^{71}$. Le dispositif qui fut ainsi créé assurait à la formation professionnelle une bien meilleure coordination que la loi de 1959 n'avait pu faire à la promotion sociale.

\section{Le financement}

Le fonds de la formation professionnelle et de la promotion sociale, qui se substituait au fonds de la promotion sociale, était alimenté par une dotation budgétaire annuelle au moins égale au produit de la taxe d'apprentissage versé au Trésor : il s'agissait donc de la seule partie de la taxe qui ne faisait pas, de la part des employeurs, l'objet d'une imputation à des dépenses effectivement faites par eux à des fins de formation. L'exposé des motifs et l'avis du Conseil économique et social retenaient une évaluation de 200 millions de francs. Il ne s'agissait toutefois que d'un minimum garanti. En 1967, 
la dotation du fonds fut de 196,60 $\mathrm{MF}^{72}$. Au fil des années, la dotation du chapitre connut une progression sensible.

D'autre part, la décision d'harmoniser la rémunération des stagiaires en application de la loi du 31 décembre 1968 s'accompagna de la création du chapitre 43-04 nouveau dans le budget de 1970 doté de 356,70 MF. La taxe d'apprentissage due au titre de l'année 1966 vit son taux passer de 0,4 à $0,6 \%$, ce qui permit un accroissement des moyens utilisés par les entreprises, et si elles ne les utilisaient pas intégralement, mis à la disposition du fonds. Les deux milliards de francs d'investissements publics pour 1967, 1968 et 1969, permirent la création de CET (55000 places), d'IUT (60 000 places), d'établissements d'enseignement agricole courts (collèges agricoles, centres de formation professionnelle et de promotion sociale agricole), mais aussi de deux instituts de promotion sociale agricole préparant au brevet de technicien et d'agent technique agricoles, aux centres de formation professionnelle de l'AFPA dont la capacité devrait passer de 56000 à 70000 places. Cette programmation traduisait non un apport nouveau de crédits, mais le financement sur trois ans de projets prévus pour une période de quatre ans.

En définitive, la mise en place du financement des nouvelles lois a bien sûr reposé initialement sur l'utilisation de moyens existants ou sur des financements déjà décidés, mais il y a eu indéniablement dès l'origine un effort constant pour accroître la dotation du fonds d'une manière significative.

\section{L'aménagement des droits individuels}

Des dispositions diverses aménagèrent certains secteurs de la formation: congé de formation non rémunéré; procédure de règlement des conflits relatifs à l'attribution de ces congés; prêts pour les personnes qui ne pouvaient bénéficier de bourses ou d'allocations ; allocations pour les mères de trois enfants et les veuves; les agriculteurs pouvaient suivre des stages de formation ou de promotion, ou des cycles de préparation à l'enseignement supérieur agronomique et des centres artisanaux de promotion et de qualification permettaient aux artisans de se préparer à la direction des entreprises artisanales avec l'aide du Fonds.

La loi du 3 décembre 1966 ne traitait que de congés sans rémunération. Ce fut la loi du 31 décembre $1968^{73}$ qui régla le problème de la rémunération, fondamental pour la réussite $d u$ projet. Elle intervint après que $M$. Debré eut quitté le ministère de l'Économie et des Finances, mais il est utile de l'évoquer, car elle constitue un élément important du dispositif de formation continue de 1966. Dans une note confidentielle du 27 avril 1967, relative à la mise en œuvre de la loi du 3 décembre 1966 ${ }^{74}$, P. Laurent mentionne parmi les travaux engagés l'unification des systèmes d'indemnisation : une étude confidentielle fut confiée à Jacques Delors, chargé de mission au Commissariat du Plan. Le rapport préparé par Jacques Delors et Guy Métais, daté du mois d'août 1968, avait pour objectif de simplifier, établir l'équité, inciter, ne pas dissuader de l'École ${ }^{75}$. Sa préparation avait ainsi été engagée avant les événements de mai 1968, qui ne furent donc pas à l'origine des dispositions de la loi de décembre mais l'ont néanmoins favorisée $^{76}$. La loi fixait un niveau de rémunération selon le type de stage par référence soit au dernier salaire perçu, soit au salaire minimum et prévoyait une prise en charge totale ou partielle par l'État. 


\section{Dispositions diverses} concernaient, dans le domaine de la Fonction publique, la création des instituts régionaux d'administration pour le recrutement et la formation des fonctionnaires de catégorie A, dans celui de l'Agriculture, la création des stages de formation et de promotion au sein des centres de formation professionnelle ou de promotion sociale agricole, dans un institut national et des instituts régionaux. Dans le domaine de la gestion, était créée la Fondation nationale pour l'enseignement de la gestion des entreprises (FNEGE) ${ }^{77}$. La formation à la gestion était un élément essentiel pour le développement économique. L'assemblée générale du Conseil d'État avait estimé devoir disjoindre les articles concernant l'enseignement agricole et la FNEGE qui ne relevaient pas du domaine législatif. Le gouvernement les reprit néanmoins dans le texte soumis au Parlement.

\section{Mise en œuvre et réalisations}

60 La loi du 3 décembre ne rencontra pas d'hostilité majeure. Le Conseil économique et social regretta que les organisations professionnelles, pourtant sollicitées pour les actions de formation, n'aient pas le même statut au niveau de la coordination que les administrations et soient reléguées dans un rôle de donneur d'avis. Ses autres observations portèrent principalement sur la pérennité de la dotation du fonds, le montant des crédits programmés, la promotion collective. À l'Assemblée nationale, des craintes furent exprimées sur les risques d'un prélèvement excessif sur la taxe d'apprentissage.

61 Le CNPF était d'accord avec l'essentiel de la loi : dans un rapport du 14 juin $1966^{78}$, donc postérieur à la décision de préparer une loi, l'assemblée générale de cet organisme constatait que la réforme de l'enseignement ne produirait ses effets que dans six ans et demandait que des mesures fussent prises entre-temps. Le CNPF demandait notamment que la FPA fût mise en mesure d'accélérer ses formations grâce à un système de conventions, que des mesures du même ordre fussent prises pour le recrutement des jeunes sortant du système scolaire, que les crédits budgétaires permettant de financer les conventions fussent accrus, que la procédure d'utilisation de la taxe d'apprentissage fût accélérée. La CGT, au contraire était hostile à la loi, y voyant un transfert au patronat des responsabilités de l'État en matière de formation, notamment par la généralisation des conventions ${ }^{79}$. Le Conseil supérieur de l'Éducation nationale émit un avis défavorable au projet; le secrétaire général de la Fédération de l'Éducation nationale, M. Lauré, lui reprochait la précipitation de la consultation, le simple accompagnement d'une réforme de l'enseignement qui procédait à une orientation précoce des élèves, et l'absence de mesure en faveur de l'enseignement technique long ${ }^{80}$.

62 Au cours de l'année 1967, les décrets d'application de la loi furent publiés. Une circulaire du Premier ministre du $1^{\mathrm{er}}$ janvier 1967 aux préfets de région mit en place des groupes régionaux permanents. Un fonctionnaire fut choisi par le préfet pour animer le groupe. Il était assisté par l'inspecteur chargé de l'échelon régional de l'éducation professionnelle (EREP) et par l'inspecteur du travail chargé de l'échelon régional de l'emploi. 
63 À l'échelon central, le secrétaire général du ministère de l'Éducation nationale, délégué du Premier ministre, détenait la réalité du pouvoir. Le discours tenu le 8 octobre 1967 à Nancy à l'occasion de la fête annuelle du travail organisée par la Société industrielle de l'Est ${ }^{81}$ explicita ses intentions. Il affirmait que "réorganisation de l'enseignement et politique de développement de la formation professionnelle s'appuient et se complètent l'une l'autre; et c'est vraisemblablement l'un des aspects les plus novateurs de l'ensemble des réformes entreprises en ces matières au cours de ces dernières années en ce qui concerne aussi bien les structures et le contenu des enseignements que leur rôle social » qu'en conjuguant la réforme de l'enseignement et l'organisation progressive de l'éducation permanente, il soit possible de «faire reculer l'encyclopédisme étouffant des programmes scolaires et universitaires "; il définissait les conditions que requérait cette éducation permanente : dispositif permanent d'étude dépassant le court terme des emplois et des formations ; définition d'une pédagogie des adultes et de méthodes de validation des acquis; mobilisation de tous les moyens d'information et de formation disponibles. C'était donc une politique globale de la formation et de l'emploi qui était ébauchée, mais elle n'eut pas le temps de se réaliser.

Les archives de la formation professionnelle permettent d'évaluer à 470000 environ le nombre des stagiaires en $1970^{82}$. Si l'on rapporte ces effectifs à ceux mentionnés pour la Promotion sociale en 1965 (453 000), on constate donc une relative stagnation du nombre de personnes en formation.

65 À l'Éducation nationale, le ministère avait pris les devants en matière d'organisation en créant dès juillet les échelons régionaux de l'éducation professionnelle ${ }^{83}$ confiés à un inspecteur principal de l'enseignement technique auprès de chaque recteur. C'est dans le cadre de la loi de 1966 que fut expérimenté en 1967 le système des «unités professionnelles » d'où sont issues par la suite les unités capitalisables, à partir des travaux menés en Meurthe-et-Moselle par le CUCES, sous le pilotage de Bertrand Schwartz, et avec la participation active d'Alain Élie, ingénieur civil des mines, en faveur de mineurs de fer, en vue de les préparer au CAP d'électrotechnique ou de construction mécanique. Ce système permit d'organiser la formation par unités et de concevoir la validation par contrôle continu, grâce à un jury itinérant statuant sur le rapport des formateurs et en s'appuyant sur un référentiel, répertoire des capacités requises pour acquérir le diplôme.

66 Dans l'agriculture, la loi de 1966 eut un impact considérable. Elle vint en renfort à la réalisation de la loi du 2 août 1960 et du décret du 20 juin 1961 sur l'enseignement agricole. La profession souffrait d'un déficit en matière de formation. Selon une étude de 1958, seulement $5 \%$ des agriculteurs avaient reçu une formation générale ou technologique de second degré. La formation des adultes dans ce domaine était en crise $^{84}$ : elle était jusqu'alors confiée aux foyers de progrès agricole, antennes des directions des services agricoles, et de ce fait critiquée par la profession qui considérait que ces attributions lui revenaient; elle ne comportait qu'une éducation gestuelle d'ailleurs fort appréciée des agriculteurs. Après le départ d'Edgard Pisani et son remplacement par Edgar Faure (6 janvier 1966), les directions des services agricoles furent supprimées, et les foyers de progrès périclitèrent. Les ingénieurs des travaux agricoles qui y étaient employés se trouvèrent sans affectation. C'est dans ce contexte que la loi du 3 décembre 1966 vint créer des centres de formation professionnelle ou de promotion agricole, en même temps qu'un institut national et des instituts régionaux de promotion. La création de l'Institut national de promotion supérieure agricole 
(INPSA) de Dijon fut l'instrument d'une véritable formation professionnelle. La conception de cette formation a été confiée à un groupe de travail piloté par Paul Moati, ingénieur du GREF. Les circulaires des 18 janvier et 21 juin 1966 ont mis en place une formation d'adultes apportant des compléments de connaissances générales, techniques et économiques et sanctionnée par le brevet professionnel agricole délivré en contrôle continu. La préparation du BPA s'effectuait dans les centres de formation professionnelle et de promotion sociale agricole. L'opération a réussi malgré la méfiance des organismes professionnels et l'indifférence de la population concernée, grâce à la détermination des fonctionnaires et au succès initial rencontré auprès de jeunes agriculteurs ayant une attitude positive à l'égard de la formation. Dès 1967, les crédits nécessaires à la mise en place de 250 CFPPA ont été obtenus. Par la suite, cette opération a été valorisée par la politique communautaire qui a lié, à partir de 1969, l'octroi de certaines aides à l'installation à une formation de 200 heures : une unité de BPA correspondait précisément à ce critère. Ensuite, certains agriculteurs ont continué leur formation pour obtenir le BPA complet. Le nombre de BPA obtenus a atteint 1500 en 1975 et 4000 en 1980. Les CFPPA ont fourni une contribution importante à la politique de formation agricole. Ils constituèrent dorénavant un élément permanent de chaque lycée agricole.

Les événements de 1968 ont eu pour conséquence le passage de M. Debré au ministère des Affaires étrangères ; d'autre part, le secrétaire général du ministère, dont le départ avait été annoncé aux recteurs par le ministre Alain Peyrefitte le 26 avril pour prendre effet le $15 \mathrm{mai}^{85}$, mais qui était resté en service du fait des événements, a quitté ses fonctions avec l'arrivée d'Edgar Faure.

Le nouveau ministre, qui s'efforçait de pacifier les rapports avec les enseignants, notamment dans l'Université, ne mettait pas la formation professionnelle au centre de ses préoccupations; il retira pratiquement l'Éducation nationale de la politique coordonnée ${ }^{86}$. Le chantier de l'application de la loi de 1966 fut repris par J. Delors. On entre dès lors dans l'histoire des lois de 1971, qu'il n'est pas envisagé de traiter ici de manière exhaustive, sauf pour mentionner que la loi sur la formation professionnelle de 1971 reprendra quasi intégralement le dispositif institutionnel de 1966, ainsi que les principales règles posées à cette époque (politique coordonnée, régime des conventions). On conçoit donc que $\mathrm{M}$. Debré ait ressenti quelque amertume à voir publier une loi qui reprenait l'essentiel de celle de $1966^{87}$, surtout après qu'il eut fait connaître son désaccord au Premier ministre de l'époque, J. Chaban-Delmas. Mais la loi de 1971 modifia considérablement le dispositif de 1966 : la création d'une taxe de 1,1\% sur les salaires pour assurer la formation du personnel en place, ce qui créa un marché de la formation, le projet de formation d'entreprise, le congé individuel de formation. L'importance des sommes dégagées par la nouvelle taxe fut une innovation de taille par rapport à la loi de 1966. Par ailleurs, la rédaction d'un nouveau texte accompagnée de l'abrogation de l'ancien est une technique juridique fréquente. L'incident montre en tout cas le sentiment de paternité dont M. Debré entourait toutes ses interventions en matière de formation.

En définitive, il y a bien eu, de 1959 à 1966, puis à 1971, un processus de création continu d'un dispositif de formation professionnelle en France. Ce dispositif a été caractérisé par sa structure interministérielle et la mise en place d'une coordination avec les organisations professionnelles. Le cadre institutionnel de la loi n'a jamais été remis en cause depuis 1959. Les avantages consentis aux bénéficiaires se sont affermis 
progressivement sans vraiment atteindre le niveau d'un véritable droit qui puisse être revendiqué devant les tribunaux. Les problèmes de coordination et de pilotage demeurent centraux et malheureusement mal résolus. Le rattachement du dispositif de pilotage au Premier ministre est un procédé fragile, à la merci de l'intérêt que celui-ci porte personnellement à la question. Le positionnement institutionnel de l'autorité à laquelle le Premier ministre délègue ses pouvoirs est aussi capital : dans le dispositif de 1966, la délégation du Premier ministre au responsable administratif du principal ministère permettait de faire avancer de conserve formation professionnelle initiale et formation professionnelle continue ; le temps a manqué pour qu'il soit possible de juger ce qu'aurait pu être une coordination forte des deux volets de la formation professionnelle. Dans le dispositif de 1971, l'importance donnée à la formation en entreprise et l'implication limitée du principal ministère formateur ont réactivé la césure entre formation initiale et formation continue des adultes. Le passage progressif des institutions interministérielles sous le pouvoir du ministre chargé du travail dans les années 1980, aggravé par la fusion des organigrammes et l'intégration du personnel, n'a fait qu'accentuer le phénomène.

70 Il est indéniable que la passion de M. Debré pour les problèmes de formation a été un des éléments moteurs de l'évolution du système de formation français depuis 1959. La formation lui paraissait être un levier essentiel pour faire évoluer la société dans le cadre d'un régime républicain, certes autoritaire, auquel il croyait sincèrement. Cette passion l'a rendu sensible à l'évolution des « besoins » de la société et de l'économie tels que les concevaient certains managers et certains responsables de formation. Ses interventions de 1959 et de 1966 ont été rendues possibles parce que la formation était un domaine que le Président de la République a accepté de laisser à son initiative particulière et parce qu'il avait le goût personnel de s'investir dans des domaines dépassant les frontières ministérielles. Il a mis fin au quasi-monopole des services publics (enseignement technique, FPA) pour faire place à un système fondé sur la coopération des services publics avec les organisations professionnelles, mais en conservant néanmoins à l'État de grands pouvoirs d'initiative, de pilotage et de contrôle, confortés par la part prépondérante faite au financement public sous forme budgétaire. Ce n'est qu'à partir de 1971 que le secteur privé bénéficiera d'un financement autonome. On peut relever la similitude de la démarche suivie en matière de formation professionnelle avec celle qui a inspiré la politique en matière d'enseignement privé : coopération des organismes privés avec l'État dans le cadre d'orientations définies par celui-ci, utilisation d'une pratique contractuelle, prise en charge du financement. Si ce dispositif a assez bien fonctionné pour renforcer la qualification de la main-d'œuvre, en revanche, les mesures prises en vue de favoriser la mobilité sociale sont, pour la plupart, restées d'une efficacité réduite, probablement par suite d'une valorisation excessive de la formation initiale dans la culture nationale. La deuxième chance est restée un vœu pieux, sans doute parce que les pouvoirs publics n'ont jamais réussi à articuler formation initiale et formation continue, ni tenté avec détermination d'y parvenir.

71 Pourtant, il subsiste une importante demande de formation promotionnelle individuelle, comme en témoignent les inscriptions au CNAM, dans les structures universitaires ouvertes aux travailleurs actifs, ou encore dans les organismes associatifs divers. Un nombre de 400000 ou 500000 personnes est souvent avancé, alors que les congés individuels de formation plafonnent à environ trente mille. Les perspectives ouvertes par la validation des acquis professionnels ou par ceux de l'expérience 
favoriseront probablement les démarches de promotion individuelle mais ne supprimeront pas les problèmes de formation, qu'elles peuvent même aviver. La formation continue à vocation promotionnelle reste donc un problème actuel. Enfin, les difficultés de la coordination inter-institutionnelle demeurent. Les expériences faites en 1959, 1966 et 1971 mériteraient une étude critique attentive: le problème institutionnel a toujours été et demeure fondamental.

\section{NOTES}

1. M. Debré : Trois républiques pour une France. Mémoires I, Paris, Albin Michel, 1984, p. 74-75.

2. Patrick Samuel : Michel Debré, l'architecte du général, Paris, Arnaud Franel Éditions, 1999, pp. 31-36.

3. M. Debré : Gouverner. Mémoires III, Paris, Albin Michel, 1988, p. 97.

4. M. Debré : Gouverner autrement. Mémoires IV, Paris, Albin Michel, 1993, p. 230

5. M. Debré : L'artisanat, classe sociale, la notion d'artisan, la législation artisanale. Thèse pour le doctorat, Paris, Dalloz, 1934. M. Debré s'appuie sur les travaux de Halbwachs. La classe se définit comme une communauté d'intérêts économiques, le rôle dans la production paraissant l'élément essentiel, et par une communauté de sentiments, qui crée une conscience et une discipline.

6. Archives Debré, FNSP, 1 DE 22.

7. Ibid.

8. Le Figaro du 10 janvier 1959 annonce 26 nouvelles ordonnances au J.O., et celui du 26 février, 36.

9. Le Figaro, et 28 février, Le Monde, 2 juillet, L'Humanité, 4 novembre 1959.

10. A. Bayard: La formation professionnelle des adultes dans les esprits et dans la loi, Montreuil, AFPA, 1970.

11. AN, F 17 bis, 9005/1.

12. J. A. Vigean : «Problèmes de la promotion du travail », L'Enseignement technique, $\mathrm{n}^{\circ}$ 15, 1957, pp. 51-86.

13. Jacques Wolff : La promotion du travail, Paris, Commissariat général à la productivité, 1958, cité par Yves Palazzeschi : Introduction à une sociologie de la formation, Paris, Montréal, L'Harmattan, 1968, vol. 2, pp. 21-30.

14. Vincent Troger : «Les passeurs de l'éducation populaire à la formation continue ", Travail et emploi, $n^{\circ} 86$, avril 2001, pp. 9-23.

15. Antoine Prost : «Jalons pour une histoire de la formation professionnelle en France », Travail et emploi, ${ }^{\circ} 86$, avril 2001, p. 6.

16. Jean Fombonne : L'affirmation de la fonction personnel dans les entreprises (France 1830-1990). Thèse d'histoire, EHESS, 2000.

17. Bulletin du CNPF, $\mathrm{n}^{\circ}$ 141, décembre 1955.

18. JORF du 6 août 1959.

19. M. Debré : Mémoires III, op. cit., pp. 97-107.

20. Entretien avec Bertrand Schwartz, le 5 novembre 2001.

21. Odile Rudelle: "La promotion sociale" inLa politique sociale du général de Gaulle, sous la direction de Marc Sadoun, Jean-François Sirinelli, Robert Vandenbussche, Villeneuve d'Ascq, PUL, 1990.

22. Entretien avec André Fanton, le 17 janvier 2002. 
23. Ces réactions sont extraites d'articles parus dans Liaisons sociales, $\mathrm{n}^{\circ}$ 55-60, supplément, 19 mai 1960, rapportées par Yves Palazzeschi : Introduction à une sociologie de la formation, Anthologie de textes français 1944-1994, volume 2, Paris, Montréal, L'Harmattan, 1998, pp. 43-53.

24. Joanine Roy : «L'aide gouvernementale à la formation des syndicalistes ", Le Monde, 16, 17 et 18 juillet 1964.

25. L'Humanité,21 avril et 14 mai 1964.

26. Jean-Charles Asselain : Le budget de l'Éducation nationale 1952-1967, Paris, PUF, 1967, p. 247.

27. AN, F 17 bis, 9005/2.

28. AN, F 17 bis, 9005/4.

29. Entretien avec M. Vatier, les 17 avril et 25 octobre 2001.

30. M. Debré : Gouverner. Mémoires III, op. cit., p. 96.

31. André Bayard : La Formation professionnelle des adultes, op. cit., p. 197.

32. Bernard Bonnet: La formation professionnelle des adultes, une institution et ses agents 1934-1994. Thèse de doctorat de sociologie, Université de Paris X Nanterre, 1994.

33. Jean-François Chosson : Les générations du développement rural 1945-1990, Paris, LGDJ, 1990.

34. AN, F 60 bis 2832 .

35. Jean-Pascal Renaix : La promotion sociale, Peuple et Culture, Paris, 1967.

36. AN, F 60 bis 2853.

37. AN, F 60 bis 2837.

38. Philippe Casella : «La promotion sociale comme forme d'intervention publique : le cas de Grenoble (1960-1966) », Travail et emploi, $\mathrm{n}^{\circ} 86,2001$, p. 49.

39. AN, 80 AJ 424.

40. Françoise Laot : La formation des adultes. Histoire d'une utopie en acte. Le complexe de Nancy, Paris, Montréal, L'Harmattan, 1999.

41. À hauteur de $435125 \mathrm{~F}$ pour un projet d'un montant de $6787611 \mathrm{~F}$; AN, F 60 bis 2837.

42. JORF débats parlementaires Sénat, séance du 27 octobre 1966, p. 1351.

43. Rapport de M. Cartailler, proviseur du lycée technique du Perreux à la suite du séminaire sur la promotion sociale de 1962, AN, F 17 bis 9005.

44. Cf. circulaire du Premier ministre du 14 mars 1962, AN, F60 bis 2832.

45. Entretien avec Jean-François Chosson le 9 novembre 2001.

46. Entretien avec Dominique Alunni le 6 mars 2002. Cf. également Joseph Rovan: Mémoires d'un Français qui se souvient d'avoir été Allemand, Paris, Seuil, 1999, p. 327.

47. Claude Dubar et Charles Gadéa (éd) : La promotion sociale en France, Paris, Presses universitaires du Septentrion, Villeneuve d'Ascq, 1999.

48. Yves Palazzeschi, op. cit., vol 2, p. 161.

49. Annuaire rétrospectif de la France 1948-1988, Paris, INSEE, 1990, p. 247.

50. Entretien avec A. Dupont-Fauville du 22 juin 2001, propos confirmés le 28 novembre 2002.

51. J.-M. Jeanneney : Une mémoire républicaine. Entretiens avec J. Lacouture, Paris, Seuil, 1997, p. 211.

52. Entretiens des 22 juin 2001 et 28 novembre 2002 précités.

53. Entretien avec D. Alunni, le 6 mars 2002.

54. H. Mendras : La seconde révolution française, Paris, Gallimard, 1988, cité par Y. Palazzeschi : Introduction àune sociologie de la formation, vol. 1, Paris, L'Harmattan, 1999, p. 167.

55. V. Troger : «Les passeurs de l'éducation populaire à la formation continue », Travail et Emploi, $\mathrm{n}^{\circ} 86$, avril 2001, p. 9.

56. M. Debré : Gouverner autrement. Mémoires IV, Paris, Albin Michel, 1993, p. 114.

57. FNSP, archives Debré, carton 4 DE, formation professionnelle 1966-1967.

58. Déclarations de Guy Métais à la journée d'étude sur les lois de 1971 organisée par le Groupe d'étude « Histoire de la formation des adultes » (GEHFA) le 29 mars 2001.

59. Ibid. et Marie-Emmanuelle Chessel et Fabienne Pavis : Le technocrate, le patron et le professeur, Paris, Berlin, 2001, p. 26. 
60. FNSP, archives Debré, carton 4 DE.

61. Note pour François Ortoli FM/GS du 15 avril 1966, AN, 80 AJ 424.

62. Archives Debré, carton 4 DE, formation professionnelle 1966-1967, note de Françoise Duléry du 14 septembre 1966.

63. M. Debré : Mémoires IV, op. cit., p. 116.

64. AN, 19990026, art. 126 / 295-437.

65. JORF Débats parlementaires Sénat,27 octobre 1966, p. 1351.

66. Entretiens avec Pierre Laurent les 6 mai 1994 et 14 novembre 2001.

67. M. Debré : Mémoires IV, op. cit., p. 114.

68. Le Moniteur, $\mathrm{n}^{\circ} 37 \mathrm{du} 10$ septembre 1966.

69. Archives privées. Note personnelle d'un membre du cabinet Debré.

70. Ibid.

71. FNSP, archives Debré, carton DE Finances 1966-1967 (services du Premier ministre, chapitre 43-03).

72. Les dotations mentionnées sont tirées des projets de lois de finances, annexes services votés mesures nouvelles, services du Premier ministre, I services généraux.

73. JOdu 3 janvier 1969.

74. AN, 80 AJ 424 .

75. AN, F 60 bis 2900.

76. Entretien avec Guy Métais, le $1{ }^{\mathrm{er}}$ juin 1993.

77. Cf. Marie-Emmanuelle Chessel et Fabienne Pavis : Le technocrate, le patron et le professeur. Une histoire de l'enseignementsupérieur de gestion, Paris, Belin, 2001.

78. Le Moniteur,28 juillet 1966.

79. Claude Michel : La CGT et l'enseignement, Institut CGT d'histoire sociale, 1990, p. 124.

80. Le Monde, 25 \& 26 septembre 1966.

81. Le Moniteur, octobre 1967.

82. AN, F 60 bis 2905.

83. Circulaire du 4 juillet 1966.

84. Jean-François Chosson : «L'odyssée des centres de formation professionnelle et de promotion agricoles ", Éducation permanente, $\mathrm{n}^{\circ}$ 77, 1984, pp. 35-43. Paul Moati : ingénieur général honoraire du GREF : «L'avènement de la formation professionnelle agricole 1966-1967 », document manuscrit.

85. AN, 198701 91-34.

86. Intervention de Guy Métais, chargé de mission au Commissariat général du plan de 1965 à 1969, à la journée consacrée à la loi de 1971 par le Groupe d'étude pour l'histoire de la formation des adultes (GEHFA) le 27 novembre 2001.

87. M. Debré : Mémoires IV, op. cit., p. 118.

\section{RÉSUMÉS}

Michel Debré a marqué l'histoire de la formation professionnelle, en 1959, par deux lois concernant la promotion sociale et la formation des militants syndicaux, puis en 1966, en instituant par la loi les structures qui constituent encore l'architecture du système français de formation des adultes. Il était inspiré par le souci d'assurer, par la promotion professionnelle, la 
cohésion nationale dont il avait constaté les carences dans l'immédiat avant-guerre.

Il a mis à profit, dans cette entreprise, un espace de liberté d'intervention politique que lui laissait le chef de l'État malgré la pression qu'exerçaient les grands problèmes politiques du moment. Par cette série de lois, il s'est trouvé à l'origine d'un processus de création continu d'un dispositif concerté avec les organisations professionnelles, qui n'a cessé de se développer malgré la complexité de son organisation interministérielle et la difficulté d'assurer aux bénéficiaires un véritable droit à la formation.

Michel Debré added his contribution to the history of vocational training through two 1959 laws which concerned social advancement and the training of trade union activists. In 1966 he also set up by law the present-day structure of the French system of adult training.

He was concerned about ensuring - through professional advancement - the unity of the nation which had before the war appeared frail to him. In his venture he made the most of the little room left by the head of government for political intervention and carried out his project despite the pressure of the major political issues of the time. Through this series of laws, he found himself at the root of a continuing process aimed at devising a new system with professional organizations. This system is still evolving in spite of the complexity of its interdepartmental organization and the difficulty in ensuring that those entitled to receive training were in fact able to take advantage of it.

Michel Debré hat die Geschichte der beruflichen Fortbildung (entscheidend) geprägt, zunächst 1959 durch zwei von ihm veranlasste Gesetze zur sozialen Förderung / Sicherung sowie zur Ausbildung der Gewerkschaftsmitglieder, und dann 1966 durch die gesetzliche Festlegung der Strukturen, die noch heute das französische System der Erwachsenenbildung prägen. Er strebte danach, den nationalen Zusammenhalt der Gesellschaft, der seines Erachtens in der Vorkriegszeit mit Mängeln behaftet war, durch eine (allgemeine) berufliche Förderung aller Arbeitnehmer zu sichern.

Bei diesem Vorhaben nutzte er weitgehend den Handlungsspielraum, den ihm der Staatspräsident trotz der damaligen politisch angespannten Situation ließ. Er initiierte durch diese Gesetze eine kontinuierliche Zusammenarbeit mit den Sozialpartnern, trotz späterer interministerieller Abstimmungsschwierigkeiten und den Problemen, den Begünstigten den Anspruch auf eine angemessene Fortbildung zu sichern.

\section{INDEX}

Index géographique : France

Mots-clés : Debré (Michel), formation des adultes, formation professionnelle, législation et réglementation

Index chronologique : XXe siècle (après 1958), XXe siècle

\section{AUTEUR}

\section{PIERRE BENOIST}

Cour des Comptes

Chercheur associé au Service d'histoire de l'éducation 